\title{
Energy Efficiency of Hybrid-Power HetNets: A Population-like Games
}

\section{Approach}

\author{
L. A. Fletscher, Member, IEEE, J. Barreiro-Gomez, C. Ocampo-Martinez, Senior Member, IEEE, \\ J. M. Maestre, Member, IEEE, and C. Valencia Peroni
}

\begin{abstract}
In this paper, a distributed control scheme based on population games is proposed. The controller is in charge of dealing with the energy consumption problem in a Heterogeneous Cellular Network (HetNet) powered by hybrid energy sources (grid and renewable energy) while guaranteeing appropriate quality of service $(\mathrm{QoS})$ level at the same time. Unlike the conventional approach in population games, it considers both atomicity and non-anonymity. Simulation results show that the proposed population-games approach reduces grid consumption by up to about $12 \%$ compared to the traditional best-signal level association policy.
\end{abstract}

\section{INTRODUCTION}

The energy efficiency in the next generation cellular networks is a field of special interest today, particularly with the exponential growth of users expected in 5G systems. This phenomenon has motivated different projects focused on the study of ways to reduce grid consumption in cellular networks. One of the conclusions of these projects is that most of the grid consumption in cellular networks is caused by base stations (BSs) and also depends on the traffic load [4]. This problem is quite related to the design of the userBS association algorithm, which determines how the network uses its resources to serve the users.

Among different alternatives to improve the energy efficiency in cellular networks [19], the utilization of renewable energies (RE) as the power source for BSs has been relevant in the last few years. However, the integration of RE in next-generation cellular networks (NGCN) presents various challenges. Some of them are related with the need for

This work was supported in part by the Colombian funding entity Departamento Administrativo de Ciencia, Tecnología e Innovación - COLCIENCIAS for the Ph.D. scholarship number 6172. Second author gratefully acknowledges support from U.S. Air Force Office of Scientific Research under grant number FA9550-17-1-0259. Also by DEOCS project (ref. DPI2016-76493-C3-3-R) from the Spanish Ministry of Culture and Sports and AGAUR and the Agència de Gestió d'Ajuts Universitaris i de Recerca of the Generalitat de Catalunya. Financial support by the Spanish MINECO project DPI2017-86918-R is also gratefully acknowledged.

L. Fletscher is with Departamento de Ingeniería Electrónica y Telecomunicaciones, Facultad de Ingeniería, Universidad de Antioquia UdeA, Medellín, Colombia (e-mail: luis.fletscher@udea.edu.co).

J. Barreiro-Gomez is with Learning \& Game Theory Laboratory, New York University Abu Dhabi, United Arab Emirates (e-mail: jbarreiro@nyu.edu).

C. Ocampo-Martinez is with the Automatic Control Department, Universitat Politècnica de Catalunya, Institut de Robòtica i Informàtica Industrial (CSIC - UPC), Barcelona, Spain (e-mail: cocampo@iri.upc.edu).

J. Maestre is with System Engineering and Automation Department, School of Engineering, Universidad de Sevilla, Seville, Spain (e-mail: pepemaestre@us.es).

C. Valencia Peroni is with Process and Energy Department, Universidad Nacional de Colombia, Medellín, Colombia (e-mail: cavalenciapa@unal.edu.co). control strategies and short-time response to guarantee the network stability despite the variability of renewable sources [9]. This is an appropriate scenario for the application of distributed control solutions [6], [16].

Game theory has been used to solve the user-BS association problem. In [3], the user-BS association problem in HetNets has been modelled as a non-cooperative game and solved with a distributed algorithm inspired by machine learning techniques. In [11], the network-selection problem has been studied by using coalitional games with an evolutionary perspective. In particular, the need for a users-centric paradigm in fully-distributed environments and the multiobjective characteristics of next generation network systems was stated. Finally, an introduction to distributed population dynamics applied to optimization and control problems can be found in [5].

In this paper, population games are used to develop a distributed user-BS association mechanism to reduce grid consumption in a HetNet powered with hybrid energy sources. To evaluate the proposed mechanisms, we utilize a two tier HetNet where the first tier is a Macro-Base station (MBS) powered by on-grid energy and the second tier is composed by small cell-base stations (SCBSs) powered only by renewable energy. This network architecture requires more demanding control strategies to guarantee quality of service (QoS) levels. Wind is the only green-energy source considered because it is highly fluctuating, fact that has a significant effect on user-BS association dynamics increasing the control requirements.

The main contribution of this paper is a novel distributed user-BS association scheme based on population games to reduce grid consumption in HetNets powered by hybrid sources without storage systems. In particular, characteristics of atomicity and non-anonymity are considered to take into account that one user decision affects the global performance of the system. It is important to note that atomicity and nonanonymity are novel features of the population games approach proposed being this the main difference with previous works as presented in [5], [17]. For this reason, we refer to the proposed mechanism as population-like games.

The remainder of the paper is as follows. In section II, the problem statement is described. Section III presents the atomicity and non-anonymity approaches in population games. Section IV describes the simulation scenario. In Section V, the performance of the proposed schemes is evaluated, including the analysis of results. Finally, in Section VI, conclusions are drawn. A summary of the notation used on 
TABLE I

NOTATION

\begin{tabular}{cc}
\hline Parameter & Description \\
\hline $\mathcal{B}$ & Set of base stations \\
$b$ & Number of base stations \\
$\ell$ & Base station's index \\
$\mathcal{U}$ & Set of users \\
$u$ & Number of users \\
$i$ & User's index \\
$p$ & Possible location \\
$k$ & Discrete time step \\
$N$ & Simulation horizon \\
$\mathcal{B}_{k}$ & Active base stations in a time $k$ \\
$\mathcal{B}_{i, k}$ & Available BSs providing service to $i \in \mathcal{U}$ at $k$ \\
$C_{\ell, k}$ & Energy consumption of BS $\ell$ at $k$ \\
$r_{i \ell \ell}^{p}$ & Transmission rate of $i \in \mathcal{U}$, connected with a BS $\ell$ at $k$ \\
$\psi_{i \ell}^{p}$ & SINR perceived by $i \in \mathcal{U}$ in $p$ from BS $\ell$ \\
$\varphi$ & Threshold: minimum SINR required to have service \\
$y_{i \ell}$ & User-BS association indicator for user $i$ with BS $\ell$ \\
$z_{A, k}$ & Number of active users at $k$ \\
$z_{\ell}^{\text {max }}$ & Users that can be served by a SCBS $\ell$ simultaneously \\
$f_{i \ell, k}$ & Fitness function perceived for $i \in \mathcal{U}$ from BS $\ell$ at $k$ \\
$\varrho_{i, k}^{h \ell}$ & Switching rule from strategy $\ell$ to strategy $h$ for $i \in \mathcal{U}$ \\
\hline
\end{tabular}

this work can be found in Table I.

\section{PROBLEM STATEMENT}

The major sources of energy consumption in a cellular network are base stations (BSs), whose consumption depends on the number of active users in a given time instant [4]. Hence, a suitable user-BS association mechanism is key to reduce on-grid consumption. To evaluate the proposed population-like games user-BS association mechanism, a two-tier downlink HetNet,which is composed of one MBS and multiple SCBSs, is used. The MBS is always on and is powered by on-grid energy, while the SCBSs are powered exclusively by renewable energy without a battery system.

Let us define a geographical area $\mathcal{A} \subset \mathbb{R}^{2}$ where base stations and users are located. The set of $b \in \mathbb{Z}_{>0}$ base stations is denoted by $\mathcal{B}=\{1, \ldots, b\}$ and a set of $u \in \mathbb{Z}_{>0}$ users is denoted by $\mathcal{U}=\{1, \ldots, u\}$. Let $p \in \mathcal{A}$ denote a possible location, and $b=1 \in \mathcal{B}$ represent the MBS. Let $k \in \mathbb{Z}_{\geq 0}$ denote the discrete time with a sampling time given by $\tau \in \mathbb{Z}_{>0}$ seconds, and let $N \in \mathbb{Z}_{>0}$ be a simulation horizon. Each SCBS updates its cell size every $\tau$ seconds by changing the transmission power according to the amount of renewable energy available at its location. In each time instant $k$, a set of $\mathcal{B}_{i, k} \subset \mathcal{B}$ base stations are available to provide service to user $i \in \mathcal{U}$.

\section{A. Energy Model}

The energy consumption model used in this paper has been proposed by project EARTH and has been widely used in works related to energy efficiency in cellular networks [8], [13], [20]. According to EARTH, the energy consumption of a BS consists of two parts: the static power consumption and the dynamic power consumption [4]. The energy consumption can be expressed as

$$
C_{\ell, k}=\Delta_{\ell} \delta_{\ell, k} T_{\ell, k}+E_{\ell}^{S}, \forall \ell \in \mathcal{B}
$$

where $\Delta_{\ell}$ is the slope of load-dependent energy consumption of $\mathrm{BS} \ell$, the transmission power of $\mathrm{BS} \ell$ at the $k^{\text {th }}$ time instant is $T_{\ell, k}$, the traffic load of BS $\ell$ at the $k^{\text {th }}$ time instant is $\delta_{\ell, k}$, and $E_{\ell}^{S}$ is the static energy consumption of $\mathrm{BS} \ell$ in each time instant. Static power consumption is related to the energy required for the normal operation of a BS, and the dynamic power consumption is the additional energy demand caused by the traffic load, which is approximated by a linear function of the load.

Here, the total energy consumption of the network scenario in a given time instant is the sum of the grid consumption (due to MBS) and the green consumption (due to SCBSs). Hence, the reduction of consumption in BS $\ell=1$ (MBS) is the key to improve energy efficiency.

\section{B. Traffic Model}

Traffic requests are modelled as an inhomogeneous Poisson point process [12]. The traffic size, the arrival rate per area $\lambda^{p} \in \mathbb{R}_{\geq 0}$, and the average traffic size $\mu^{p} \in \mathbb{R}_{\geq 0}$, for all $p \in \mathcal{A}$, are independently distributed. Moreover, these three elements are used in order to capture the spatial traffic variability, as in [12].

A mobile user $i \in \mathcal{U}$, at location $p \in \mathcal{A}$, associated with a BS $\ell \in \mathcal{B}_{i, k}$, has a transmission rate denoted by $r_{i \ell}^{p} \in \mathbb{R}_{\geq 0}$, which can be generally expressed according to the ShannonHartley theorem [12] as

$$
r_{i \ell}^{p}=W_{\ell} \cdot \log _{2}\left(1+\psi_{i \ell}^{p}\right), \quad \forall i \in \mathcal{U}, \ell \in \mathcal{B}_{i, k}, p \in \mathcal{A},
$$

where $W_{\ell} \in \mathbb{R}_{\geq 0}$, is the operating bandwidth. The received signal by user $i \in \mathcal{U}$ at location $p \in \mathcal{A}$ from $\ell \in \mathcal{B}_{i, k}$ is given by signal-to-interference-plus-noise ratio (SINR) denoted in this paper by $\psi_{i \ell}^{p} \in \mathbb{R}_{\geq 0}$.

In this work, it is assumed that the network's frequency scheduling is such that each SCBS can only serve a fixed number $z_{\ell}^{\max }$ of users simultaneously for all $\ell \in \mathcal{B} \backslash\{1\}$. Nevertheless, the MBS has no limit for the number of served users, being able to serve all active users at an instant $z_{A, k}$. Note that the bandwidth assigned to a user $i \in \mathcal{U}$ connected to the $\mathrm{BS} \ell \in \mathcal{B}_{i, k}$ is affected by the number of users connected to it for the channels available must be shared between the active users.

Assuming that mobile users are uniformly distributed in the coverage area of all BSs, the traffic load of the $\ell^{\text {th }}$ BS in the $k^{\text {th }}$ time instant can be expressed as

$$
\begin{aligned}
\delta_{\ell, k} & =\frac{\sum_{i \in \mathcal{U}} y_{i \ell, k}}{U_{\ell, k}}, \forall \ell \in \mathcal{B}, \\
U_{\ell, k} & = \begin{cases}z_{A, k}, & \text { if } \ell=1, \\
z_{\ell}^{\max }, & \text { otherwise, }\end{cases}
\end{aligned}
$$

where $y_{i \ell}$ is the user association indicator, i.e., if user $i \in \mathcal{U}$ is associated to the $\mathrm{BS} \ell \in \mathcal{B}$, then $y_{i \ell}=1$, and $y_{i \ell}=0$ otherwise. Moreover, note that $0 \leq \delta_{\ell} \leq 1$, for all $\ell \in \mathcal{B}$ and time instant $k$.

The average transmission rate per user in the $k^{\text {th }}$ time instant depends on $\psi$ and the number of users connected to 
the serving BS [2], which allows to express (2) as

$$
r_{i \ell, k}^{p}=\frac{W_{\ell}}{\sum_{i \in \mathcal{U}} y_{i \ell, k}} \log _{2}\left(1+\psi_{i \ell}^{p}\right), \forall i \in \mathcal{U}, \ell \in \mathcal{B}_{i, k} .
$$

According to [1], at each time instant, a user can be associated with the $\ell^{\text {th }} \mathrm{BS}$ if the received signal level $\psi_{i \ell}^{p}$ is greater than a threshold $\varphi$ that indicates the minimum signal level required by a user to have service.

\section{On-grid Energy Consumption Optimization Problem}

As previously mentioned, on-grid consumption reduction and adequate transmission rates are design requirements in NGCN. Hence, the optimization problem has two objectives: i) to reduce the overall system grid consumption, and ii) to maximize the average transmission rate per user. According to this, it is possible to formulate the following optimization problem:

$$
\begin{aligned}
\min _{y_{11, k}, \ldots, y_{n 1, k}} J\left(y_{11, k}, \ldots, y_{n 1, k}\right)= \\
\sum_{k=1}^{N}\left\{\gamma_{1} \sum_{i \in \mathcal{U}} y_{i 1, k}-\gamma_{2} \frac{W_{\ell}}{\sum_{i \in \mathcal{U}} y_{i \ell, k}} \log _{2}\left(1+\psi_{i \ell}^{p}\right)\right\},
\end{aligned}
$$

s.t.

$$
\begin{aligned}
\sum_{i \in \mathcal{U}} y_{i \ell, k} \leq z_{\ell}^{\max }, & \forall \ell \in \mathcal{B} \backslash\{1\}, k \in[0, N] \cap \mathbb{Z}_{\geq 0}, \\
y_{i \ell, k} . \psi_{i \ell}^{p} \geq \varphi, & \forall i \in \mathcal{U}, \ell \in \mathcal{B}_{i, k}, k \in[0, N] \cap \mathbb{Z}_{\geq 0}, \\
\sum_{\ell \in \mathcal{B}} y_{i \ell, k} \leq 1, & \forall i \in \mathcal{U}, k \in[0, N] \cap \mathbb{Z}_{\geq 0}, \\
y_{i \ell, k} & \in\{0,1\}, \quad \forall i \in \mathcal{U}, \ell \in \mathcal{B}, k \in[0, N] \cap \mathbb{Z}_{\geq 0},
\end{aligned}
$$

where (5a) is the objective function, which focuses on minimizing consumption from the grid and maximizing user's transmission rate with an optimal assignment of active users to available BSs at each time instant. Moreover, $\gamma_{1}, \gamma_{2} \in \mathbb{R}_{>0}$ are weights assigned to each objective. Expressions in (5b)(5e) are the problem constraints: (5b) establishes that a small cell $\ell$ can serve a maximum of $z_{\ell}^{\max }$ users simultaneously; (5c) is the user's received signal level constraint, (5d) requires that a user is served only by one BS in a time instant; and $(5 \mathrm{e})$ establishes that $y_{i, \ell}$ is a binary variable.

The optimization problem (5) is a multi-objective mixed integer problem (MIP), a well-known NP-hard problem [10]. However, the distributed control strategy based on population games proposed in this paper is a suitable alternative for reducing the computational burden. Improving computational burden is possible since each user solves a limited maximization problem based only on the comparison of its current fitness function with the fitness function offered by the subset of BSs $\left(\mathcal{B}_{i, k}\right)$.

Another key element in the proposed game-theory-basedmechanism is the possibility of designing a fitness function according to the cost function in the optimization problem. In this case, the fitness function maintains the weight for each objective and includes an incentive to choose a BS powered by renewable energy. These features are here expressed as

$$
f_{i \ell, k}=\gamma_{1} P_{i \ell}+\gamma_{2} \tilde{r}_{i \ell, k}^{p}, \forall \ell \in \mathcal{B}_{i, k},
$$

where the condition $\gamma_{1}+\gamma_{2}=1$ must hold. Here, $P_{i \ell}$ is the incentive received for user $i \in \mathcal{U}$ to choose a cell $\ell \in \mathcal{B}_{i}$ according to the energy source and $\tilde{r}_{i \ell, k}^{p}$ is the normalized transmission rate that can receive user $i \in \mathcal{U}$ from BS $\ell \in$ $\mathcal{B}_{i}$ at time instant $k \in[0, N] \cap \mathbb{Z}_{\geq 0}$. On-grid energy has a higher economic and environmental impact compared to green energy, being suitable to consider a network-operator policy focused on encouraging users to use cells powered by renewable energies. For this reason, in this paper it is proposed a green incentive $G$ for users such that

$$
P_{i \ell}= \begin{cases}G, & \text { if } \ell=1, \\ 2 G, & \text { if } \ell \in \mathcal{B} \backslash\{1\} .\end{cases}
$$

The energy efficiency problem is studied using a distributed population-like game approach with atomicity and non-anonymity features. Hence, in each time instant $k \in$ $[0, N]$ a user $i \in \mathcal{U}$ with revision opportunity evaluates its fitness function $f_{i \ell, k}$ among available choices $\mathcal{B}_{i, k}^{p} \subset \mathcal{B}$ and selects the destination $\mathrm{BS}$ according to the switching rule denoted by $\varrho_{i, k}^{h \ell}[18]$.

\section{ATOMICITY AND NON-ANONYMITY IN POPULATION-LIKE GAME APPROACH}

Some of the main characteristics of population dynamics, which can be seen as restrictive features for applying this game-theoretical approach in engineering applications, are the anonymity and non-atomicity [7].

Definition 1: (Anonymity [7]) The anonymity describes the situation in which the index of decision makers does not affect the utility function. This concept can also be associated with the homogeneousness of decision makers selecting strategies, i.e., decision makers are assumed to be indistinguishable within the same strategy.

Definition 2: (Atomicity [7]) The atomicity describes the situation in which a single decision maker affects the global utility.

This paper presents an alternative population-like game approach that allows dealing with atomicity and nonanonymity. In fact, it is assumed that each decision maker within the population is different, and consequently each decision maker has a different utility. Therefore, each individual decision maker affects the global utility. In addition, all the decision makers selecting the same strategy are considered to be different even though they belong to the same strategy.

Let $\mathcal{U}$ be the set of rational decision makers in a population located throughout a bi-dimensional geographical area denoted by $\mathcal{A} \subset \mathbb{R}^{2}$. These agents are rational in the sense that they make decisions pursuing the improvement of their individual benefits. Moreover, let $\mathcal{B}=\{1, \ldots, b\}$ denote the set of choices that the set of decision makers have. More precisely, let $\mathcal{B}_{i, k}^{p} \subset \mathcal{B}$ denote the possible choices that the $i^{\text {th }}$ decision maker has at time instant $k$ depending on its geographical position $p \in \mathcal{A}$, where $\mathcal{B}_{i, k}^{p} \neq \emptyset$, for all $i \in \mathcal{U}, k \in \mathbb{Z}_{>0}, p \in \mathcal{A}$. In other words, the sets $\mathcal{B}_{i}$, for all $i \in \mathcal{U}$ define possible interaction sets. For simplicity, it is omitted the superscript $p$, indicating that the set of available 
strategies for each decision maker depends on its position, i.e., $\mathcal{B}_{i, k}=\mathcal{B}_{i, k}^{p}$.

The set of decision makers selecting the strategy $\ell \in \mathcal{B}$ at time instant $k$ is given by $\mathcal{U}_{k}^{\ell} \subseteq \mathcal{U}$. Note that the cardinality $\left|\mathcal{U}_{k}^{\ell}\right|=\sum_{i \in \mathcal{U}} y_{i \ell, k}$ for all $k$ and $|\mathcal{U}|=\sum_{\ell \in \mathcal{B}} \sum_{i \in \mathcal{U}} y_{i \ell, k}$. Moreover, consider a strategic profile given by a distribution of decision makers $\mathcal{U}$ throughout the set of choices $\mathcal{B}$, i.e., $\left(\mathcal{U}_{k}^{1}, \ldots, \mathcal{U}_{k}^{b}\right)$, which represents the population state, where $\bigcap_{\ell \in \mathcal{B}} \mathcal{U}_{k}^{\ell}=\emptyset$, and $\bigcup_{\ell \in \mathcal{B}} \mathcal{U}_{k}^{\ell}=\mathcal{U}$. Also, let $g_{i}=\{\ell \in \mathcal{B}$ : $\left.i \in \mathcal{U}^{\ell}\right\}$ return the strategy that a decision maker $i \in \mathcal{U}$ is choosing. In addition, let the amount of decision makers be constrained at each possible choice, i.e., $\left|\mathcal{U}_{k}^{\ell}\right| \leq z_{\ell}^{\max }$, being $z_{\ell}^{\max } \in \mathbb{Z}_{>0}$, for all $\ell \in \mathcal{B}$.

Assumption 1: The initial distribution of decision makers $\left(\mathcal{U}_{0}^{1}, \ldots, \mathcal{U}_{0}^{b}\right)$ in the population is feasible, i.e., $\left|\mathcal{U}_{0}^{\ell}\right| \leq z_{\ell}^{\max }$, for all $\ell \in \mathcal{B}$. It implies that $n=|\mathcal{U}| \leq \sum_{\ell \in \mathcal{B}} z_{\ell}^{\max }$. Moreover, $\bigcap_{\ell \in \mathcal{B}} \mathcal{U}_{0}^{\ell}=\emptyset$, and $\bigcup_{\ell \in \mathcal{B}} \mathcal{U}_{0}^{\ell}=\mathcal{U}$.

Let $f_{i \ell, k} \in \mathbb{R}$ be the fitness function for the decision maker $i \in \mathcal{U}$ selecting the strategy $\ell \in \mathcal{B}$ at time instant $k \in \mathbb{Z}_{>0}$. If two decision makers $i, j \in \mathcal{U}$ are selecting the same strategy $\ell \in \mathcal{B}$, then $f_{i \ell, k} \neq f_{j \ell, k}$ since the population considers non-anonymity. Furthermore, since the decision maker $i \in \mathcal{U}$ cannot select the strategies $\mathcal{B} \backslash \mathcal{B}_{i, k}$, then for simplicity it is considered that the decision maker has no incentives to move to such a strategy, i.e., $f_{i \ell, k}=0$, for all $\mathcal{B} \backslash \mathcal{B}_{i, k}$. The objective within the population is to achieve a Local $\varepsilon$-Equilibrium [17] as presented in Definition 3, which also provides notions about Local Nash Equilibrium [14], [15].

Definition 3: (Local $\varepsilon$-Equilibrium). Let $\varepsilon \in \mathbb{R}_{\geq 0}$. A population distribution $\left(\mathcal{U}^{1 *}, \ldots, \mathcal{U}^{b *}\right)$ is a Local $\varepsilon$-Equilibrium with respect to the interaction sets $\mathcal{B}_{i}$ if all decision makers $i \in \mathcal{U}^{\ell *}$, for all $\ell \in \mathcal{B}$, satisfy the following condition:

$$
f_{i \ell} \geq f_{i \ell^{\prime}}-\varepsilon, \quad \forall \ell, \ell^{\prime} \in\left\{h \in \mathcal{B}_{i}:\left|\mathcal{U}^{h}\right|<z_{h}^{\max }\right\}
$$

On the other hand, if condition (8) holds with $\varepsilon=0$, then $\left(\mathcal{U}^{1 *}, \ldots, \mathcal{U}^{b *}\right)$ is a Local Nash Equilibrium with respect to the interaction sets $\mathcal{B}_{i}$.

The population evolves according to switching rules, which determine the timing and the result of decision makers' choices. Let $\varrho_{i, k}^{h \ell} \in \mathbb{R}_{\geq 0}$ represent the switching rule for the $i^{\text {th }}$ decision maker. Therefore, if $\varrho_{i, k}^{h \ell}>0$, then the decision maker $i \in \mathcal{U}$ has incentives to move from the $h^{\text {th }}$ strategy to the $\ell^{\text {th }}$ at time instant $k$. The evolution of the population is made by assigning a revision opportunity as described in [18]: a decision maker is chosen randomly from the population, and it receives an opportunity to decide whether or not it should move to another strategy by comparing its utility with those it would obtain by selecting the strategy with a higher fitness function from the set $\mathcal{B}_{i, k}$.

Assumption 2: Suppose that a decision maker $i \in \mathcal{U}$ receives a revision opportunity. Then, before its next revision opportunity, all decision makers $j \in \mathcal{U} \backslash\{i\}$ receive a revision opportunity.

Being $i \in \mathcal{U}$ the decision maker with a revision opportu- nity, then the procedure is as follows:

$$
\begin{aligned}
& \mathcal{U}_{k+1}^{g_{i}}=\mathcal{U}_{k}^{g_{i}} \backslash\left\{i \operatorname{sgn}\left(\varrho_{i, k}^{g_{i} \ell}\right)\right\}, \text { for any } i \in \mathcal{U}, \\
& \mathcal{U}_{k+1}^{\ell}=\mathcal{U}_{k}^{\ell} \cup\left\{i \operatorname{sgn}\left(\varrho_{i, k}^{g_{i} \ell}\right)\right\} \backslash\{0\}, \text { for any } \ell \in \mathcal{B}_{i, k} .
\end{aligned}
$$

Notice that the equilibrium in (9) is achieved when $\varrho_{i}^{g_{i} \ell}=$ 0 , for all $i \in \mathcal{U}, \ell \in \mathcal{B}_{i}$, i.e., when the decision maker $i \in \mathcal{U}$ has not incentives to move to any strategy $\ell \in \mathcal{B}_{i, k}$. In this case, $\mathcal{U}_{k+1}^{\ell *}=\mathcal{U}_{k}^{\ell *}$, for all $\ell \in \mathcal{B}$.

Remark 1: Notice that in (9) each decision maker $i \in \mathcal{U}$ does not require full information from the population, but only local information from $\mathcal{B}_{i}$.

Now, it is necessary to define an appropriate switching rule for the population. Consider the following switching rule:

$$
\varrho_{i, k}^{h \ell}=\left(z_{\ell}^{\max }-\left|\mathcal{U}_{k}^{\ell}\right|\right) \max \left(0, f_{i \ell, k}-f_{i h, k}-\varepsilon\right), \forall h, \ell \in \mathcal{B}_{i},
$$

where $\varepsilon \in \mathbb{R}_{>0}$. Notice that the switching rule in (10) indicates that the $i^{\text {th }}$ decision maker has incentives to move from the $h^{\text {th }}$ to the $\ell^{\text {th }}$ strategy only if it represents an improvement over its utilities greater than $\varepsilon$ and there is available capacity at the $\ell^{\text {th }}$ strategy. Proposition 1 shows that an equilibrium in dynamics (9) with the aforementioned switching rule implies a Local $\varepsilon$-Equilibrium with respect to the allowed interactions within the population.

Proposition 1: ( $\varepsilon$-Equilibrium Point) The equilibrium point of the dynamics in (9) with the switching rule in (10) implies a Local $\varepsilon$-Equilibrium with respect to the interaction sets $\mathcal{B}_{i}$, for all $i \in \mathcal{U}$.

Proof: It immediately follows from the fact that the equilibrium in (9) implies that $\varrho_{i}^{g_{i} \ell}=0$, with $i \in \mathcal{U}^{g_{i} *}$, for all $i \in \mathcal{U}, \ell \in \mathcal{B}_{i}$. Therefore, $f_{i \ell, k} \leq f_{i g_{i}, k}+\varepsilon$, with $i \in \mathcal{U}^{g_{i} *}$, for all $i \in \mathcal{U}, \ell \in \mathcal{B}_{i}$ such that $z_{\ell}^{\max }<\left|\mathcal{U}_{k}^{\ell}\right|$, which is the required conclusion according to Definition 3.

In addition to obtaining a local equilibrium (Definition 3), Proposition 2 shows the satisfaction of constraints stated involving the initial condition in Assumption 1, for all the time instants $k \in \mathbb{Z}_{>0}$.

Proposition 2: (Satisfaction of constraints) If Assumption 1 holds, then, $\left|\mathcal{U}_{k}^{\ell}\right| \leq z_{\ell}^{\max }, \bigcap_{\ell \in \mathcal{B}} \mathcal{U}_{k}^{\ell}=\emptyset$, and $\bigcup_{\ell \in \mathcal{B}} \mathcal{U}_{k}^{\ell}=\mathcal{U}$ under dynamics in (9) for all time instants $k \in \mathbb{Z}_{>0}$.

Proof: Regarding the first constraint, it is assumed that $\left|\mathcal{U}_{0}^{\ell}\right| \leq z_{\ell}^{\max }$, for all $\ell \in \mathcal{B}$. Moreover, notice that the cardinality $\left|\mathcal{U}_{k}^{\ell}\right|$ only can growth one by one, for all $\ell \in \mathcal{B}$, due to the fact $z_{\ell}^{\max } \in \mathbb{Z}_{>0}$ and that $\varrho_{i, k}^{g_{i} \ell}=0$ in (9) if constraint $\left|\mathcal{U}_{k}^{\ell}\right| \leq z_{\ell}^{\max }$ is active. Then $\left|\mathcal{U}_{k}^{\ell}\right| \leq z_{\ell}^{\max }$, for all $\ell \in \mathcal{B}$ and all $k \in \mathbb{Z}_{>0}$. Regarding the second constraint, notice that $0 \notin \mathcal{U}_{k}^{\ell}$, for all $\ell \in \mathcal{B}$. It follows that if $\bigcap_{\ell \in \mathcal{B}} \mathcal{U}_{k}^{\ell}=\emptyset$, then for any $i \in \mathcal{U}, \mathcal{U}_{k}^{g_{i}} \cap \mathcal{U}_{k}^{\ell}=\emptyset$, and $\left\{\mathcal{U}_{k}^{g_{i}} \backslash \mathcal{T}\right\} \cap\left\{\mathcal{U}_{k}^{\ell} \cup \mathcal{T}\right\}=\emptyset$, for any set $\mathcal{T}$.

Regarding the third constraint:

$\begin{array}{ll}\mathcal{U}_{k+1}^{g_{i}} \cup \mathcal{U}_{k+1}^{\ell}= & \left\{\mathcal{U}_{k}^{g_{i}} \backslash\left\{i \operatorname{sgn}\left(\varrho_{i, k}^{g_{i} \ell}\right)\right\}\right\} \cup \\ \left\{\mathcal{U}_{k}^{\ell} \cup\left\{i \operatorname{sgn}\left(\varrho_{i, k}^{g_{i} \ell}\right)\right\} \backslash\{0\}\right\} & =\mathcal{U}_{k}^{g_{i}} \cup \mathcal{U}_{k}^{\ell}, \text { completing } \\ \text { the proof. } & \end{array}$

\section{CASE STUDY}

The scenario described in Section II was implemented to evaluate the proposed mechanism. The case study considered 
TABLE II

Simulation PARAMETERS

\begin{tabular}{lcc}
\hline \multicolumn{1}{c}{ Parameter } & Value & Units \\
\hline Coverage Area & 3.5 & $\mathrm{~km}^{2}$ \\
System & LTE & - \\
N. Macro Base Station & 1 & - \\
N. SCBS & 36 & - \\
Inter-site distance & 500 & $\mathrm{~m}$ \\
Tx power MBS & 43 & $\mathrm{dBm}$ \\
Tx power SCBS & 22 & $\mathrm{dBm}$ \\
Static Power Cons. MBS & 130 & $\mathrm{~W}$ \\
Static Power Cons. SCBS & 6.8 & $\mathrm{~W}$ \\
Consumption Slope MBS & 4.7 & - \\
Consumption Slope SCBS & 4.0 & - \\
Path loss between MBS and user & Cost $231 \mathrm{model}$ & - \\
Antenna Gain & 15 & $\mathrm{dBi}$ \\
Max. users simultaneously for an SCBS & 100 & - \\
Receiver sensitivity & -107.5 & $\mathrm{dBm}$ \\
Time instant length & 1 & $\mathrm{~s}$ \\
Mobility Model & Random walk point & - \\
Mobility Speed & 4 & $\mathrm{~km} / \mathrm{h}$ \\
$\gamma_{1}$ & 0.6 & - \\
$\gamma_{2}$ & 0.4 & - \\
$G$ & 0.5 & - \\
\hline
\end{tabular}

is composed of one MBS and 36 overlapping SCBSs. The MBS is powered by on-grid energy, which is always on, ensuring constant coverage over the area. Each BS covers one sector, and only large-scale loss is considered in the simulations.

From a telecommunications viewpoint, the technical parameters of the simulation are defined according to a LongTerm-Evolution system in a coverage area of $3.5 \mathrm{~km}^{2}$ [1]. The distance between BSs is $500 \mathrm{~m}$ and users are uniformly distributed in the coverage area. Users are moving according to a random walk point model with $4 \mathrm{~km} / \mathrm{h}$ speed. Table II summarizes the parameters used in the simulation. In traditional cellular networks, mobile users connect to the BS that offers the best SINR, which depends on BS power transmission, path loss, and interference from other BSs. However, this mechanism is not entirely adequate for HetNets, since SCBSs with available resources can be ignored by users when receiving a stronger signal from an MBS [2]. In the rest of the paper, this procedure is referred to as the traditional scheme, and it will be the baseline for evaluating the performance of the proposed game-theorybased mechanism. In particular, to evaluate the performance of the proposed game-theory-based mechanism, a dynamic scenario with a controlled wind profile is used to enable different groups of SCBS during specific time periods. As a consequence, the number of active BSs changes according to the pre-defined sequence.

\section{RESULTS AND DISCUSSION}

Using MATLAB $R$, it was possible to evaluate the proposed schemes and their impact on grid power consumption and users transmission rate in an environment with changing characteristics of renewable sources.

Figure 1 shows the user-BS association process in the scenario with the controlled wind and 1000 users, which changes the network topology each 60 time instants. Initially, users are connected to the MBS because it is the only station active. In time instant 61 (Figure 1(a)), a sector of small cells is activated and some users change their BS according to the revision protocol. It is important to note that the revision opportunity is a probabilistic process. Hence not all users make a decision at the same time. In Figure 1(b), it is possible to observe the game evolution at the end of this wind episode $(k=120)$. Here, the largest number of users in the area with active SCBS are distributed over green cells. It is important to note that some users continue to connect to the MBS despite being in a location with coverage of green cells, for they find a better utility in the MBS either due to the cells overload or because these users are located on the edges of small cells where the transmission rate is worse. In this case, the second element of the objective function presented in (5a) has a dominant role in the utility function.

Figure 1(c) presents the game evolution in $k=360$. Here, it is observed that most users with the possibility of accessing a green cell are associated with one of them. Finally, Figure 1(d) shows the last time instant in the period with all SCBS active. There, it is possible to observe a uniform distribution of users over green cells and the accomplishment of the objective of discharging traffic load from MBS to cells powered by renewable energies. Regarding the users connected to the MBS, besides the evaluation of utility function mentioned previously, it is important to remember that the process of user generation is dynamic and, for this reason, at each time interval there will be users who have not started the game. These new active users are connected to the base station with the best signal level, in this case the MBS.

Regarding energy efficiency, the proposed game-theorybased mechanism has a grid consumption of $79.7 \mathrm{~kW}$ compared with $91 \mathrm{~kW}$ of the traditional scheme. This represents a reduction up to $12.4 \%$. On the other hand, the average transmission rate is $17.1 \mathrm{Mbps}$ with the traditional scheme and 16.6 Mbps with the game-theory-based mechanism, which is a consequence of the priority to reduce on-grid consumption and the relationship between signal level and the user rate presented in (2). Nevertheless, the impact over network throughput is minimal $(2.4 \%)$. Figure 2 shows the behaviour of cost function components. There it is possible to observe a gradual reduction of grid consumption along the simulation time Figure 2(a). In the same way, in Figure 2(b) we can see a gradual maximization of average user rate until a stable point. This behaviour is in line with the objective function presented in $(5 a)$.

\section{CONCLUSIONS}

The goal of this paper was to study a distributed gametheory-based mechanism to control the user-BS association process in a HetNet powered by renewable energy, reducing grid consumption and improving energy efficiency. The proposed mechanism is based on a population game with characteristics of atomicity and non-anonymity, elements not considered previously in proposals based on similar methodologies. 


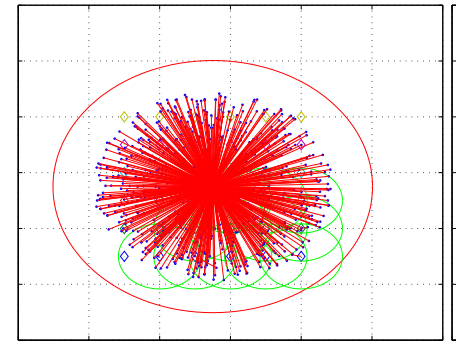

(a)

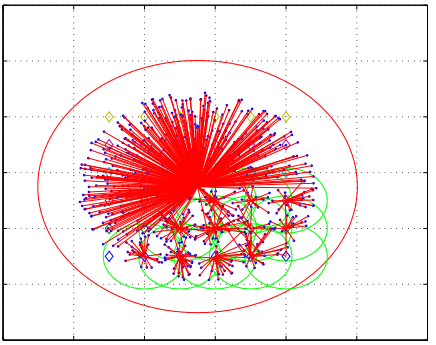

(b)

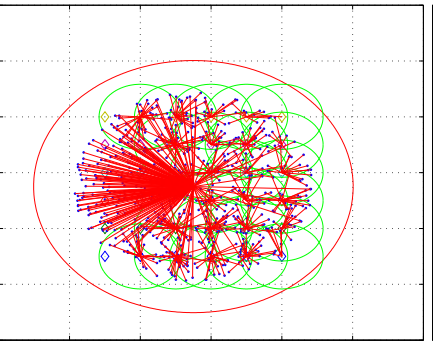

(c)

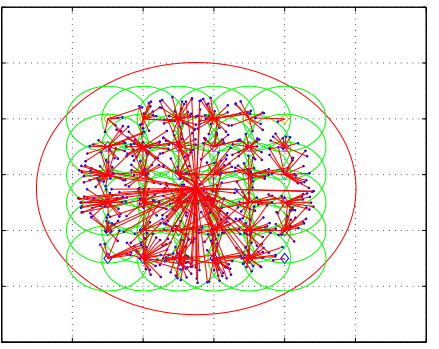

(d)

Fig. 1. User-BS association process with the proposed scheme. State of network at different time instants (a) $k=61$, (b) $k=120$, (c) $k=360$, and (d) $k=480$.

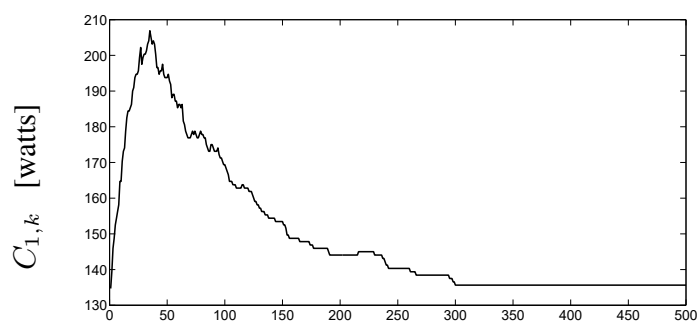

(a)

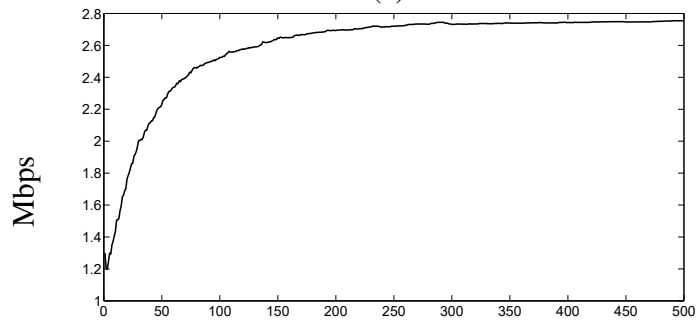

(b)

Fig. 2. Behaviour of the cost function components.

The distributed population dynamics mechanism has been shown to be a suitable option for balancing traffic in dense HetNets and reducing grid consumption through traffic discharge from MBS to green SCBS. Another important characteristic observed is the possibility to reduce the users searchspace to a subset of strategies, which facilitates solving the integer association problem, being a proper option for controlling systems with a large amount of users, as expected in next generations of cellular networks. It is part of our future investigation to formally relate the equilibrium of the proposed game-theoretical approach with the solution of the optimization problem.

Finally, it is important to emphasize that the proposed distributed game-theory-based mechanism can be used to attain other goals related to the performance of the network through the modification of the utility function.

\section{REFERENCES}

[1] 3GPP. "LTE Evolved Universal Terrestrial Radio Access Network (E-UTRAN); Self-configuring and self-optimizing network (SON) use cases and solutions", 2014. http://www.3gpp.org/ dynareport/36902.htm.

[2] J. Andrews. "Seven ways that Hetnets are a cellular paradigm shift". IEEE Communications Magazine, 51(3):136-144, 2013.

[3] A. Arani, M. Omidi, A. Mehbodniya, and F. Adachi. "A distributed learning based user association for heterogeneous networks". Trans- actions on Emerging Telecommunications Technologies, 28(5):1-13, 2017.

[4] G. Auer, V. Giannini, C. Desset, I. Gódor, P. Skillermark, M. Olsson, M. Imran, D. Sabella, M. Gonzalez, O. Blume, and A. Fehske. "How much energy is needed to run a wireless network?". IEEE Wireless Communications, 18(5):40-49, 2011.

[5] J. Barreiro-Gomez, G. Obando, and N. Quijano. "Distributed Population Dynamics: Optimization and Control Applications". IEEE Transactions on Systems, Man, and Cybernetics: Systems, 47(2):304314, 2017.

[6] E. Camponogara and H. Scherer. "Distributed Optimization for Model Predictive Control of Linear Dynamic Networks With Control-Input and Output Constraints". IEEE Transactions on Automation Science and Engineering, 8(1):233-242, 2011.

[7] B. Djehiche, A. Siwe, and H. Tembine. "Mean-Field-Type Games in Engineering". ArXiv Preprint, pages 1-22, 2016.

[8] T. Han and N. Ansari. "A Traffic Load Balancing Framework for Software-Defined Radio Access Networks Powered by Hybrid Energy Sources". IEEE/ACM Transactions on Networking, 24(2):1038-1051, 2016.

[9] H. Hassan, L. Nuaymi, and A. Pelov. "Classification of renewable energy scenarios and objectives for cellular networks". In IEEE International Symposium on Personal, Indoor and Mobile Radio Communications, PIMRC, pages 2967-2972, London, UK, 2013.

[10] D. Johnson and M. Garey. "Computers and Intractability: A Guide to the Theory of NP-Completeness". Bell Telephone Laboratories, Incorporated, New Jersey, NJ, USA, 1 edition, 1979.

[11] M. Khan and H. Tembine. "Evolutionary Coalitional Games in Network Selection". In Wireless Advanced (WiAd), pages 185-194, London, UK, 2011.

[12] H. Kim, G. de Veciana, X. Yang, and M. Venkatachalam. "Distributed $\alpha$-Optimal User Association and Cell Load Balancing in Wireless Networks". IEEE/ACM Transactions on Networking, 20(1):177-190, 2012.

[13] D. Liu, Y. Chen, K. Chai, T. Zhang, and M. Elkashlan. "Two Dimensional Optimization on User Association and Green Energy Allocation for HetNets with Hybrid Energy Sources". IEEE Transactions on Communications, 63(11):4111-4124, 2015.

[14] J. Marden. "The role of information in multiagent coordination". In 53rd IEEE Conference on Decision and Control (CDC), pages 445450, Los Angeles, CA, USA, 2014.

[15] J. Marden. "The Role of Information in Distributed Resource Allocation". IEEE Transactions on Control of Network Systems, 4(3):654$664,2016$.

[16] R. Negenborn and J. Maestre. "Distributed Model Predictive Control: An Overview and Roadmap of Future Research Opportunities". IEEE Control Systems Magazine, 34(4):87-97, 2014.

[17] G. Obando, J. Barreiro-Gomez, and N. Quijano. "A class of population dynamics for reaching epsilon-equilibria: Engineering applications". In American Control Conference (ACC), 2016, pages 4713-4718, Boston, MA, USA, 2016.

[18] W. Sandholm. "Population Games and Evolutionary Dynamics". MIT Press., Cambridge, MA, USA, 1 edition, 2010.

[19] L. Suarez, L. Nuaymi, and J. Bonnin. "An overview and classification of research approaches in green wireless networks". EURASIP Journal on Wireless Communications and Networking, 2012(1):142, 2012.

[20] H. Yang, G. Geraci, and T. Quek. "Energy-Efficient Design of MIMO Heterogeneous Networks With Wireless Backhaul". IEEE Transactions on Wireless Communications, 15(7):4914-4927, 2016. 